PROCEEDINGS OF THE

AMERICAN MATHEMATICAL SOCIETY

Volume 135, Number 8, August 2007, Pages 2555-2565

S 0002-9939(07)08749-7

Article electronically published on February 6, 2007

\title{
ON THE REVERSIBLE QUADRATIC CENTERS WITH MONOTONIC PERIOD FUNCTION
}

\author{
J. VILLADELPRAT \\ (Communicated by Carmen C. Chicone)
}

\begin{abstract}
This paper is devoted to studying the period function of the quadratic reversible centers. In this context the interesting stratum is the family of the so-called Loud's dehomogenized systems, namely

$$
\left\{\begin{array}{l}
\dot{x}=-y+x y \\
\dot{y}=x+D x^{2}+F y^{2}
\end{array}\right.
$$

We determine several regions in the parameter plane for which the corresponding center has a monotonic period function. To this end we first show that any of these systems can be brought by means of a coordinate transformation to a potential system. Then we apply a monotonicity criterium of R. Schaaf.
\end{abstract}

\section{Introduction AND STATEMENT OF THE RESUlt}

The present paper deals with the period function of the quadratic centers. The literature has used different terminology to classify these centers but essentially there are four families: Hamiltonian, reversible $Q_{3}^{R}$, codimension four $Q_{4}$ and generalized Lotka-Volterra systems $Q_{3}^{L V}$. According to Chicone's conjecture [1] the reversible centers have at most two critical periods, and the centers of the three other families have a monotonic period function. In fact there is much analytic evidence that the conjecture is true. Indeed, Coppel and Gavrilov [7] proved that the period function of any Hamiltonian quadratic center is monotonous and, more recently, Zhao 23. showed that the codimension four centers have the same property. Concerning the $Q_{3}^{L V}$ centers there are very few results. In the middle $80 \mathrm{~s}$ several authors [13, 16, 21] showed independently the monotonicity of the classical LotkaVolterra centers (which constitute a hypersurface inside the $Q_{3}^{L V}$ family), and more recently the same property has been proved in 20] for two other hypersurfaces.

From the point of view of the study of the period function it is clear therefore that the most interesting family of centers is the reversible one. By an affine transformation and a constant rescaling of time (see 26] for instance), any reversible quadratic center can be brought to Loud's normal form

$$
\left\{\begin{array}{l}
\dot{x}=-y+B x y \\
\dot{y}=x+D x^{2}+F y^{2}
\end{array}\right.
$$

Received by the editors March 13, 2006 and, in revised form, April 11, 2006.

2000 Mathematics Subject Classification. Primary 34C07, 34C25.

The author was partially supported by CONACIT through grant 2001SGR-00173 and by DGES through grant MTM2005-06098-C02-1. 


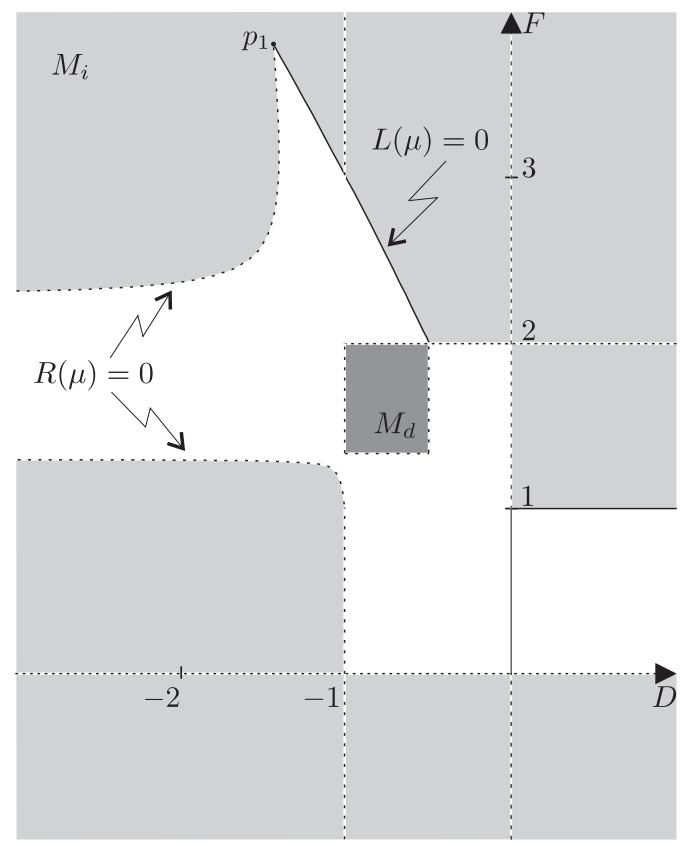

FiguRE 1. Monotonicity regions according to Theorem $\mathrm{A}$.

It is proved in 9 that if $B=0$, then the period function of the center at the origin of the above system is monotonous increasing. The remaining cases, namely $B \neq 0$, can be brought with a rescaling to $B=1$, i.e.

$$
\left\{\begin{array}{l}
\dot{x}=-y+x y, \\
\dot{y}=x+D x^{2}+F y^{2} .
\end{array}\right.
$$

As the excellent paper of Chicone and Jacobs [4] shows, all the interesting phenomena concerning the period function of quadratic centers occur in this family. This is precisely the family of quadratic centers that we study and, following the terminology in [4, we call them dehomogenized Loud's systems. The bifurcation diagram of the period function of these centers is studied extensively in [12]. Among other results, the authors determine several regions in the parameter plane for which the corresponding center has at least one critical period (and so its period function is not monotonous). In this paper we focus on the opposite situation, namely the parameters that give rise to a center with a monotonous period function. In short, we find regions of monotonicity in the parameter plane.

Theorem $\mathrm{A}$ is our main result and in its statement we refer to some regions in the parameter plane of the dehomogenized Loud's systems (11). To be more precise, see Figure 1, $M_{i}$ stands for the union of the light grey regions and $M_{d}$ is the rectangle $\left(-1,-\frac{1}{2}\right) \times\left(\frac{4}{3}, 2\right)$ in dark grey. The functions $R(\mu)$ and $L(\mu)$ used to define some boundary components of $M_{i}$ are introduced in (7) and (9) respectively. Here and in what follows we use the notation $\mu:=(D, F)$ for the sake of shortness. In particular the point $p_{1}$ is approximately $\mu=(-1.44,3.81)$. We prove the following result: 


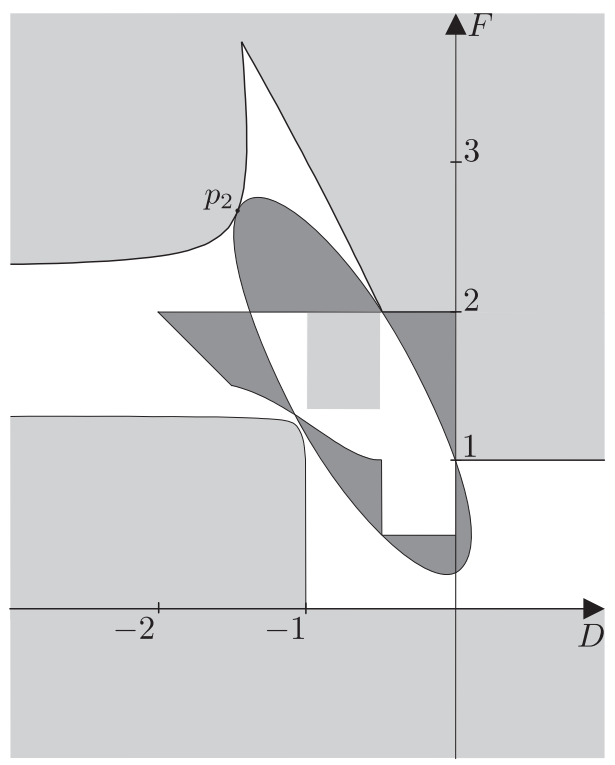

FiguRE 2. Regions of monotonicity (light grey) and regions of non-monotonicity (dark grey).

Theorem A. The period function of the center at the origin of system (11) is monotonous increasing (respectively, decreasing) in the case that $\mu \in M_{i}$ (respectively, $\left.\mu \in M_{d}\right)$.

At this point it is interesting to locate the monotonicity regions $M_{d}$ and $M_{i}$ in Theorem $\mathrm{A}$ with respect to the regions of non-monotonicity established by Theorem 5.1 in [12. This is done in Figure 2, where the ellipse that constitutes several boundary components of the regions of non-monotonicity is given by $10 D^{2}+10 D F-D+4 F^{2}-5 F+1=0$. The intersection point $p_{2}$ is approximately $\mu=(-1.47,2.66)$.

There are two other results on the dehomogenized Loud's systems that should be referred to. Thus, by using a Picard-Fuchs approach, Y. Zhao can completely describe the behaviour of the period function in two straight lines of the parameter plane, namely $F=3 / 2$ in [24] and $F=2$ in 25]. There is a number of different authors that have treated the general question of monotonicity of the period function (see [2, 3, 8, 14, 17, 22] and references there in). Apart from the monotonicity problem, several other questions related to the behaviour of the period function have been extensively studied. Let us quote, for instance, the isochronicity problem (see [5, 6, 10]) and the bifurcation of critical periods (see [11, 15, 19]).

Note added in proof. After this paper was accepted we knew about the publication of [R. Chouikha, Monotonicity of the period function for some planar differential systems. Part I: Conservative and quadratic systems, Appl. Math. (Warsaw) 32 (2005), 305-325. MR2213617]. In that paper the author shows the monotonicity for the parameters in the straight lines $F+2 D=1$ and $F=-1$ and some segments inside $D=-1 / 2, D=0, F=1$ and $F=2$. 


\section{Definitions AND PROOF OF THE RESUlt}

A critical point $p$ of a planar differential system is a center if it has a punctured neighbourhood that consists entirely of periodic orbits surrounding $p$. The largest punctured neighbourhood with this property is called the period annulus of the center and, in what follows, it will be denoted by $\mathcal{P}$. The period function of the center assigns to each periodic orbit in $\mathcal{P}$ its period. We say that the period function of a center is monotonous increasing (respectively, decreasing) if for any pair of periodic orbits inside $\mathcal{P}$, say $\gamma_{1}$ and $\gamma_{2}$ with $\gamma_{1} \subset \operatorname{Int}\left(\gamma_{2}\right)$, we have that the period of $\gamma_{2}$ is greater (respectively, smaller) than the one of $\gamma_{1}$. (Here by $\operatorname{Int}(\gamma)$ we mean the bounded connected component of $\mathbb{R}^{2} \backslash\{\gamma\}$.)

If $V$ is a smooth function with $V^{\prime}(0)=0$ and $V^{\prime \prime}(0)>0$, then the potential system

$$
\left\{\begin{array}{l}
\dot{u}=-v \\
\dot{v}=V^{\prime}(u)
\end{array}\right.
$$

has a center at the origin. Let $\mathcal{I}$ denote the projection of its period annulus on the $u$-axis. R. Schaaf proves in [17] the following monotonicity criterium:

Theorem 2.1 (Schaaf's criterium). The center at the origin of system (2) has a monotonous increasing period function in the case that

$$
\left(I_{1}\right) \quad 5 V^{\prime \prime \prime}(u)^{2}-3 V^{\prime \prime}(u) V^{(4)}(u)>0 \text { for any } u \in \mathcal{I} \text { with } V^{\prime \prime}(u)>0
$$

and

$$
\left(I_{2}\right) \quad V^{\prime}(u) V^{\prime \prime \prime}(u)<0 \text { for any } u \in \mathcal{I} \text { with } V^{\prime \prime}(u)=0 .
$$

On the other hand, in the case that

$$
\text { (D) } 5 V^{\prime \prime \prime}(u)^{2}-3 V^{\prime \prime}(u) V^{(4)}(u)<0 \text { for any } u \in \mathcal{I} \text { with } V^{\prime \prime}(u) \geqslant 0,
$$

then the period function is monotonous decreasing.

Although (11) is not a potential system, the following result will allow us to apply Schaaf's criterium to study the period function of its center.

Lemma 2.2. If $F \neq 0$, then the coordinate transformation $\left\{u=\frac{(1-x)^{-F}-1}{F}\right.$, $\left.v=(1-x)^{-F} y\right\}$ brings (1) to the potential system

$$
\left\{\begin{array}{l}
\dot{u}=-v \\
\dot{v}=D(F u+1)\left((F u+1)^{\frac{-1}{F}}-1\right)\left((F u+1)^{-\frac{1}{F}}-\frac{D+1}{D}\right) .
\end{array}\right.
$$

Proof. An easy computation shows that the change of variables given in the statement brings (11) to (3). However, for the sake of completeness, let us say a few words about how we obtain it. In short, if a system with a non-degenerated center has a first integral which is quadratic in $y$ and the corresponding integrating factor depends only on $x$, then Lemma 4.1 in [9] shows that it can be brought to a potential system and it also provides the coordinate transformation that achieves it. One can check that system (11) verifies these conditions. For instance if $F \notin\{0,1 / 2,1\}$, then $H(x, y)=(1-x)^{-2 F}\left(\frac{1}{2} y^{2}+q(x)\right)$, where

$$
q(x):=\frac{D}{2(F-1)} x^{2}-\frac{D-F+1}{(F-1)(2 F-1)} x+\frac{D-F+1}{2 F(F-1)(2 F-1)}
$$


is a first integral and $f(x)=(1-x)^{-2 F-1}$ is its corresponding integrating factor (see [18]).

Note that system (1) has $\{x=1\}$ as an invariant straight line and so the period annulus of the center at the origin is contained in the half-plane $\{x<1\}$. Accordingly the coordinate transformation in Lemma 2.2 is well defined on the whole period annulus.

On the other hand if $H(u, v)=\frac{1}{2} v^{2}+V(u)$ is the Hamiltonian of system (3), then one can check that

$$
\begin{aligned}
& V^{\prime}(u)=D(F u+1)\left((F u+1)^{\frac{-1}{F}}-1\right)\left((F u+1)^{-\frac{1}{F}}-\frac{D+1}{D}\right), \\
& V^{\prime \prime}(u)=-(2 D+1)(F-1)(F u+1)^{\frac{-1}{F}}+D(F-2)(F u+1)^{\frac{-2}{F}}+F(D+1), \\
& V^{\prime \prime \prime}(u)=(2 D+1)(F-1)(F u+1)^{\frac{-1}{F}-1}-2 D(F-2)(F u+1)^{\frac{-2}{F}-1}, \\
& V^{(4)}(u)=-(2 D+1)\left(F^{2}-1\right)(F u+1)^{\frac{-1}{F}}-2+2 D\left(F^{2}-4\right)(F u+1)^{\frac{-2}{F}-2} .
\end{aligned}
$$

Remark 2.3. The key point to succeed in applying Schaaf's monotonicity criterium to our problem was that, setting $z=(F u+1)^{\frac{-1}{F}}$, from (5) we have that

$$
V^{\prime}(u)=(F u+1) V_{1}(z), V^{\prime \prime}(u)=V_{2}(z), V^{\prime \prime \prime}(u)=\frac{V_{3}(z)}{F u+1}, V^{(4)}(u)=\frac{V_{4}(z)}{(F u+1)^{2}}
$$

for some polynomial $V_{i}$. For instance, it is clear that $V_{2}(z):=D(F-2) z^{2}-$ $(2 D+1)(F-1) z+F(D+1)$. Taking this into account it follows that

$$
5 V^{\prime \prime \prime}(u)^{2}-3 V^{\prime \prime}(u) V^{(4)}(u)=\frac{5 V_{3}(z)^{2}-3 V_{2}(z) V_{4}(z)}{(F u+1)^{2}}
$$

and observe that the numerator of the last expression is polynomial in $z$ as well. More concretely one can check that $5 V_{3}(z)^{2}-3 V_{2}(z) V_{4}(z)=z S(z)$, where

$$
\begin{aligned}
S(z):= & -2 D^{2}(3 F-4)(F-2)^{2} z^{3}+D(9 F-5)(F-1)(F-2)(2 D+1) z^{2} \\
& +\frac{1}{2}\left(\left(F^{2}(16-9 F)-2 F+4\right)(2 D+1)^{2}+3 F\left(F^{2}-4\right)\right) z \\
& +3 F(D+1)(2 D+1)\left(F^{2}-1\right) .
\end{aligned}
$$

This fact simplifies the application of Schaaf's criterium because it enables us to verify its conditions by using polynomial functions in $z$. For instance, since $z=$ $(F u+1)^{\frac{-1}{F}}$ is positive, condition $I_{1}$ is equivalent to showing that $S(z)>0$ for any $z=(F u+1)^{\frac{-1}{F}}$ with $u \in \mathcal{I}$ and $V_{2}(z)>0$.

For the sake of convenience we shall first study the parameters that fulfill condition $I_{2}$. Henceforth we use the notation

$$
\kappa(\mu):=(F-1)^{2}+(2 D+1)^{2}-1 .
$$

The ellipse $\kappa(\mu)=0$ divides the rectangle $[-1,0] \times[0,2]$ into five connected components; see Figure 3 . The sets $A_{1}$ and $A_{2}$ in the following result are two of these components.

Lemma 2.4. If $\mu \notin A_{1} \cup A_{2}$ and $F D(F-2)(D+1) \kappa(\mu) \neq 0$, then the center at the origin of system (3) verifies condition $I_{2}$ of Schaaf's criterium. 

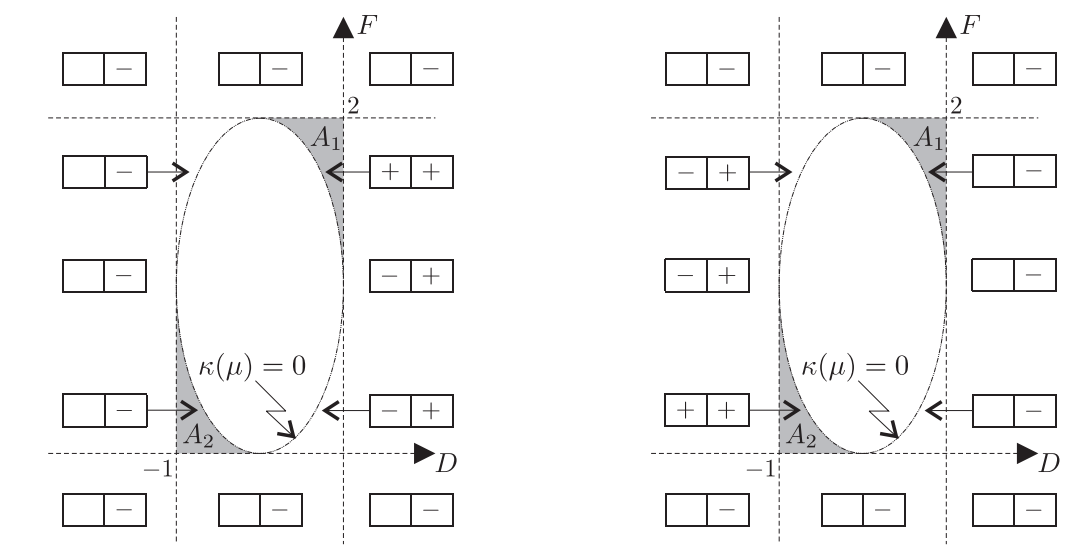

sign of $z_{+}><\operatorname{sign}$ of $V_{1}\left(z_{+}\right) V_{3}\left(z_{+}\right) \quad \operatorname{sign}$ of $z_{-} \longrightarrow<\operatorname{sign}$ of $V_{1}\left(z_{-}\right) V_{3}\left(z_{-}\right)$

Figure 3. Study for the proof of Lemma 2.4.

Proof. Note that $V^{\prime}(u) V^{\prime \prime \prime}(u)$ is polynomial in $z$ because, from (6), we have that $V^{\prime}(u) V^{\prime \prime \prime}(u)=V_{1}(z) V_{3}(z)$. In addition, $V^{\prime \prime}(u)=V_{2}(z)$. Thus, since $z=(F u+1)^{\frac{-1}{F}}$ is positive, it suffices to determine those parameters such that $V_{1}(z) V_{3}(z)<0$ for any $z>0$ with $V_{2}(z)=0$. One can check that $V_{2}(z)=0$ if and only if

$$
z=z_{ \pm}:=\frac{(2 D+1)(F-1) \pm \sqrt{\kappa(\mu)}}{2 D(F-2)} .
$$

To compute the sign of $V_{1}\left(z_{ \pm}\right) V_{3}\left(z_{ \pm}\right)$at any parameter $\mu$ we take advantage of the resultant between the polynomials $V_{2}(z)$ and $V_{1}(z) V_{3}(z)$, which is equal to $F D^{2}(F-2)(D+1)^{2} \kappa(\mu)$. It is clear then that $V_{1}\left(z_{ \pm}\right) V_{3}\left(z_{ \pm}\right)$vanishes if and only if $F D(F-2)(D+1) \kappa(\mu)=0$. This curve splits up the parameter plane into 13 connected components. By continuity, in each of these components the sign of $V_{1}\left(z_{ \pm}\right) V_{3}\left(z_{ \pm}\right)$does not change, and so it is enough to compute it in 13 concrete parameters. We proceeded in this way to obtain Figure 3, which gathers all the information needed to show the result. For instance condition $I_{2}$ is verified in the strip $(0,+\infty) \times(0,2)$ although $V_{1}\left(z_{+}\right) V_{3}\left(z_{+}\right)>0$ because $z_{+}$is negative there. Let us mention finally that the region $\kappa(\mu)<0$ is included as well because $z_{ \pm}$are not real for these parameters.

Next we shall study which parameters verify condition $I_{1}$. This is done in Lemma 2.5. and to prove it we use the bifurcation diagram of the graphics of $V_{2}$ and $S$ (recall Remark 2.3). To be more precise, we need the distribution of the roots and their location with respect to $z=0$. The distribution of roots will provide us the necessary information about the sign of $V_{2}$ and $S$. We are also interested in their location with respect to $z=0$ because $z=(F u+1)^{\frac{-1}{2}}$ is positive. The leading coefficient of $V_{2}$ and $S$ vanishes at

$$
D(F-2)=0 \text { and } D(3 F-4)(F-2)=0
$$

respectively. On the other hand, the independent term of $V_{2}$ and $S$ becomes zero at

$$
F(D+1)=0 \text { and } F\left(F^{2}-1\right)(D+1)(2 D+1)=0
$$




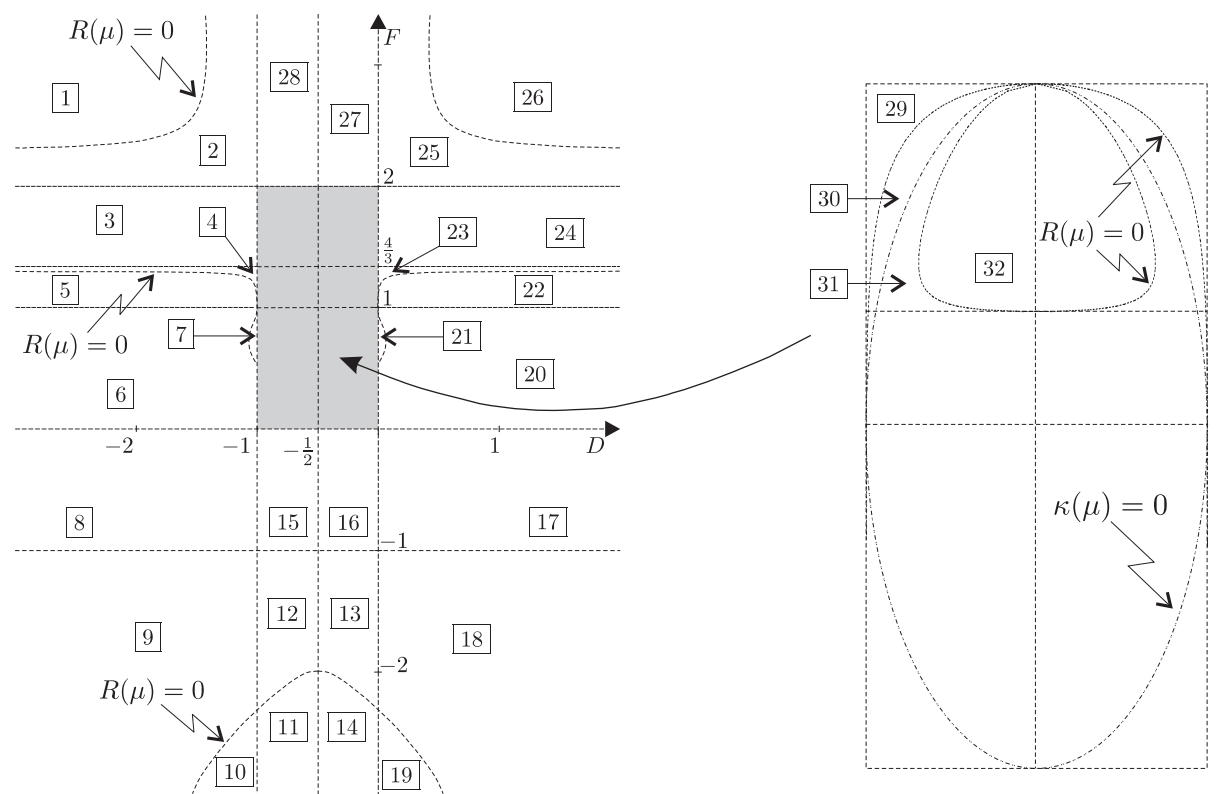

Figure 4. Bifurcation diagram of the graphics of $V_{2}$ and $S$.

respectively. The discriminant of $V_{2}$ is $\kappa(\mu)$ and the one of $S$ is $D^{2}(F-2)^{2} \kappa(\mu) R(\mu)$, where

$$
\begin{aligned}
R(\mu):= & -\left(745 F^{4}-2800 F^{3}+2688 F^{2}-442 F+52\right)(2 D+1)^{4} \\
& -3 F(F-2)\left(125 F^{4}-518 F^{3}+174 F^{2}+382 F-1\right)(2 D+1)^{2} \\
& +9 F^{2}(3 F-4)(F-2)^{2}(F+2)^{3} .
\end{aligned}
$$

Finally the resultant between $V_{2}$ and $S$ is $D^{2} F(D+1)(F-2)^{2} \kappa(\mu)^{2}$. It is clear therefore that the above-mentioned diagram has

$$
D F(D+1)(2 D+1)\left(F^{2}-1\right)(F-2)(3 F-4) \kappa(\mu) R(\mu)=0
$$

as a bifurcation curve. This curve splits up the parameter plane into several connected components and, as in the proof of Lemma 2.4 to obtain the bifurcation diagram it suffices to study one concrete parameter in each region. We compile all this in Figures 4 and 5. However, for the sake of brevity, inside the rectangle $[-1,0] \times[0,2]$ we only show the graphics of those parameters verifying condition $I_{1}$. This saves us having to draw $14+4=18$ graphics because, although imperceptible in Figure 4, the curve $R(\mu)=0$ goes into the square $[-1,0] \times[0,1]$ giving rise to 4 more regions. It is important to mention that this curve can be easily studied because $R$ is a second degree polynomial in $(2 D+1)^{2}$. Observe in particular that it is symmetric with respect to the straight line $D=-1 / 2$. We can now prove the following.

Lemma 2.5. The center at the origin of system (3) verifies condition $I_{1}$ of Schaaf's criterium if $\mu$ is inside one of the following open sets (see Figure (4):

(a) Regions 1, 5-8, 11-18, 22-24 and 27.

(b) Regions 9, 10, 25 and 26. 

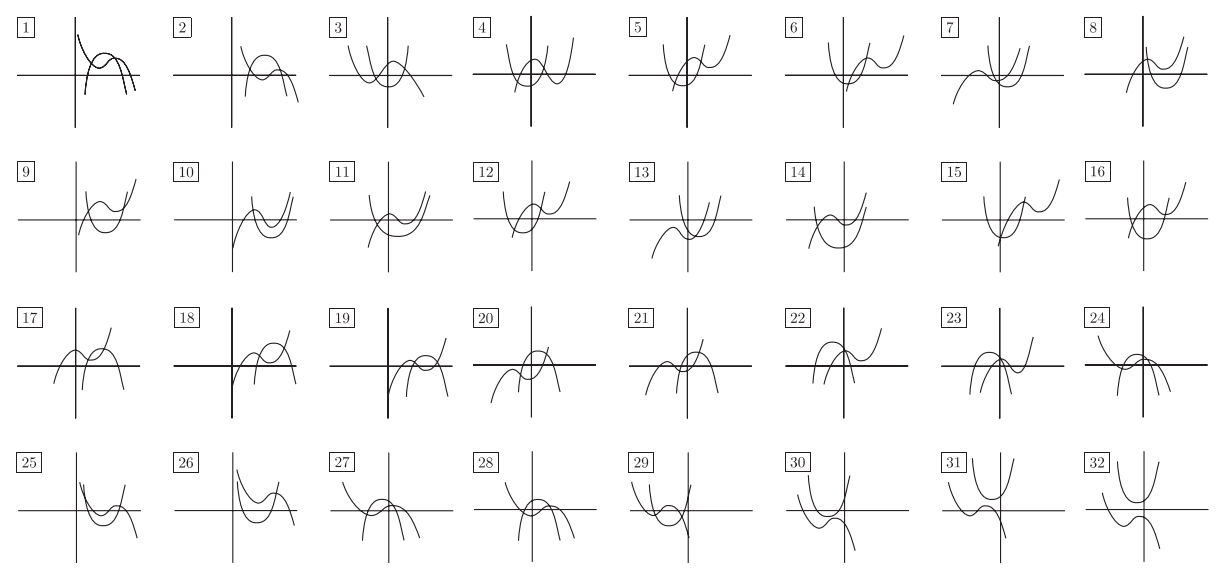

Figure 5. The graphics of $V_{2}$ and $S$ corresponding to Figure 4.

On the other hand, the center verifies condition $D$ if $\mu$ is inside the regions 29-32.

Proof. As usual let $\mathcal{I}$ denote the projection on the $u$-axis of the period annulus of the center at the origin of system (3). From Remark 2.3. the condition $I_{1}$ is verified for a parameter $\mu$ if and only if

$$
S(z)>0 \text { for all } z \text { such that } V_{2}(z)>0 \text { and } z=(F u+1)^{\frac{-1}{F}} \text { with } u \in \mathcal{I} .
$$

In view of Figure 5 , the parameters inside the regions listed in $(a)$ verify that $S(z)>0$ for all $z>0$ with $V_{2}(z)>0$. Thus, since $(F u+1)^{\frac{-1}{F}}$ is positive, it is clear that (8) holds.

Let us turn now to the parameters listed in $(b)$. A simple computation shows that if $D \notin[-1,0]$, then system (3) has a saddle at the point $\left(u^{\star}, 0\right)$, where $\left(F u^{\star}-1\right)^{\frac{-1}{F}}=$ $\frac{D+1}{D}$. Accordingly $u^{\star} \notin \mathcal{I}$. It is easy to check that $u^{\star}<0$ for $D>0$ and $u^{\star}>0$ for $D<-1$. Therefore $\mathcal{I} \subset\left(u^{\star},+\infty\right)$ for $D>0$ and $\mathcal{I} \subset\left(-\infty, u^{\star}\right)$ for $D<-1$. Thus, since $u \longmapsto(F u+1)^{\frac{-1}{F}}$ is decreasing, it suffices to verify (8) for $z \in\left(0, \frac{D+1}{D}\right)$ in the case that $D>0$ and for $z>\frac{D+1}{D}$ in the case that $D<-1$. Taking this into account, and using the fact that $V_{2}\left(\frac{D+1}{D}\right)=-\frac{D+1}{D}$ is negative for $D \notin[-1,0]$, it is clear from the graphics in Figure 5 that the parameters inside the regions listed in $(b)$ verify (8).

Finally, again from Remark 2.3, condition $D$ is satisfied for a parameter $\mu$ if and only if $S(z)<0$ for all $z$ such that $V_{2}(z) \geqslant 0$ and $z=(F u+1)^{\frac{-1}{F}}$ with $u \in \mathcal{I}$. Since for the parameters inside the regions 29-32 (see Figure 5) we have that $S(z)<0$ for all $z>0$, the result follows.

The next result is also devoted to investigating which parameters verify condition $I_{1}$. However, since the approach is different from the one in Lemma 2.5, we prefer to treat it separately. The sets $\widehat{U}_{1}$ and $\widehat{U}_{2}$ that appear in the statement are drawn in Figure 6.

Lemma 2.6. The center at the origin of system (3) verifies condition $I_{1}$ of Schaaf's criterium if $\mu \in \widehat{U}_{1} \cup \widehat{U}_{2}$. 


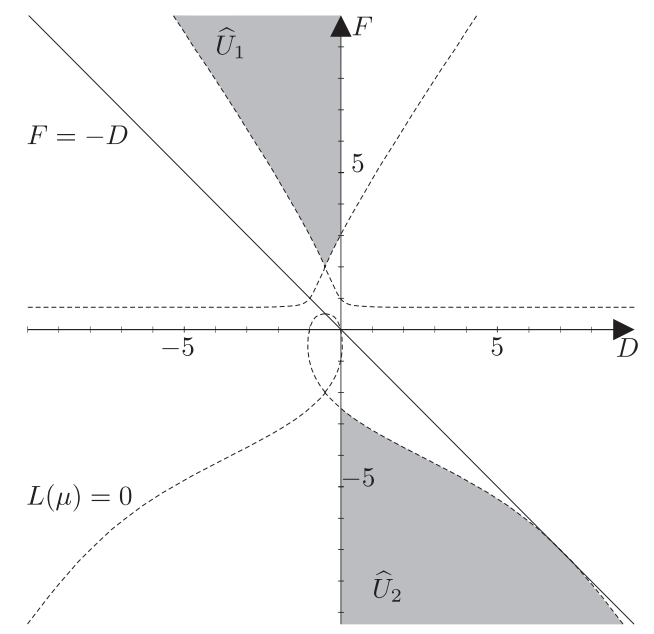

Figure 6 . The sets $\widehat{U}_{1}$ and $\widehat{U}_{2}$ in Lemma 2.6 .

Proof. In fact we will show that if $\mu \in \widehat{U}_{1} \cup \widehat{U}_{2}$, then $V^{(4)}(u)<0$ for all $u \in \mathcal{I}$, which obviously implies condition $I_{1}$. From (5) we have that

$$
V^{(4)}(u)=(F u+1)^{\frac{-1}{F}-2}\left(-(2 D+1)\left(F^{2}-1\right)+2 D\left(F^{2}-4\right)(F u+1)^{\frac{-1}{F}}\right) .
$$

Thus it is clear that the fourth derivative of $V$ only changes its sign once, at $u=u^{\star}$, where

$$
\left(F u^{\star}+1\right)^{\frac{-1}{F}}=\frac{(2 D+1)\left(F^{2}-1\right)}{2 D\left(F^{2}-4\right)} .
$$

Consequently to prove the result it suffices to verify that if $\mu \in \widehat{U}_{1} \cup \widehat{U}_{2}$, then $V^{(4)}(0)<0$ and $u^{\star} \notin \mathcal{I}$. The phase portraits of system (1) are very well known (see [12, 18, for instance). In particular, recall (4), the conic $\frac{1}{2} y^{2}+q(x)=0$ is invariant. Moreover for the parameters inside

$$
U:=\{F>1,-F<D<0\} \cup\{-F>D>0\},
$$

one can check that the conic is a hyperbola with the focal points in the $x$-axis and $q(0)<0$. Taking this into account and using the coordinate transformation in Lemma 2.2 to bring system (3) to (11), it follows that $q\left(1-(F u+1)^{\frac{-1}{F}}\right)<0$ is a necessary condition for $u \in \mathcal{I}$ in the case that $\mu \in U$. In particular, if $\mu \in U$, then $q\left(1-\left(F u^{\star}+1\right)^{\frac{-1}{F}}\right) \geqslant 0$ guarantees that $u^{\star} \notin \mathcal{I}$. Some computations show that

$$
q\left(1-\left(F u^{\star}+1\right)^{\frac{-1}{F}}\right)=\frac{\left(F^{3}+7 F^{2}+17 F-16\right)(2 D+1)^{2}-(2 F-1)\left(F^{2}-4\right)^{2}}{8 D F(2 F-1)\left(F^{2}-4\right)^{2}}
$$

and $V^{(4)}(0)=1-6 D-F^{2}$. In short, we can assert that if $\mu \in U$ verifies $6 D+F^{2}-1>0$ and $\frac{\left(F^{3}+7 F^{2}+17 F-16\right)(2 D+1)^{2}-(2 F-1)\left(F^{2}-4\right)^{2}}{D F(2 F-1)} \geqslant 0$, then $V^{(4)}(u)<0$ for all $u \in \mathcal{I}$ as desired. Straightforward arguments show that the parameters inside $U$ satisfying these two inequalities are precisely $\widehat{U}_{1} \cup \widehat{U}_{2}$, see Figure 6 where

$$
L(\mu):=\left(F^{3}+7 F^{2}+17 F-16\right)(2 D+1)^{2}-(2 F-1)\left(F^{2}-4\right)^{2} .
$$


This completes the proof of the result.

Proof of Theorem A. Thanks to the coordinate transformation in Lemma 2.2, to show the result we can consider the potential system (3). Then, by applying Schaaf's criterium, the combination of Lemmas 2.4 and 2.5 proves the result except for some parameters in the regions 2, 19 and 28 (compare Figures 11 and 4). There are parameters inside these regions that verify condition $I_{1}$, but to determine them we use Lemma 2.6 instead. To this end it is only necessary to study where the curves $L(\mu)=0$ and $R(\mu)=0$ intersect. (This is easy because $L$ and $R$ have degree one and two in $(2 D+1)^{2}$ respectively.) One can show in this way (see Figure 6) that $\widehat{U}_{2}$ contains the region 19 and that $\widehat{U}_{1}$ intersects with the region 1 as shown in Figure 1 .

Let us conclude with some technical comments about the definition of the sets $M_{i}$ and $M_{d}$ in Figure 1. Following the standard convention, Theorem $\mathrm{A}$ does not apply at those parameters in dotted curves. For instance, it is not valid at

$$
D F(D+1)(F-2)=0
$$

because Lemma 2.4 says nothing in these straight lines. This should also be the case for the parameters at $(2 D+1)(3 F-4)\left(F^{2}-1\right) R(\mu) \kappa(\mu)=0$ because Lemma 2.5 does not apply there. However an accurate analysis of the graphics of $V_{2}$ and $S$ shows that most of these parameters do verify condition $I_{1}$. Since this is rather long to explain and it does not significantly improve the result, we prefer not to prove it. However we do include these parameters in the sets $M_{i}$ and $M_{d}$ because it simplifies the statement of Theorem $\mathrm{A}$

\section{REFERENCES}

[1] C. Chicone, review of The period function of a Hamiltonian quadratic system, W. A. Coppel and L. Gavrilov, Differential Integral Equations 6 (1993). MR.1235199(94h:58072)

[2] C. Chicone, The monotonicity of the period function for planar Hamiltonian vector fields, J. Differential Equations 69 (1987), 310-321. MR0903390 (88i:58050)

[3] C. Chicone, Geometric methods of two-point nonlinear boundary value problem, J. Differential Equations 72 (1988), 360-407. MR0932371 (89e:34032)

[4] C. Chicone and M. Jacobs, Bifurcation of critical periods for plane vector fields, Trans. Amer. Math. Soc. 312 (1989), 433-486. MR0930075 (89h:58139)

[5] C. Christopher and J. Devlin, On the classification of Liénard systems with amplitudeindependent periods, J. Differential Equations 200 (2004), 1-17. MR2046315 (2004m:34067)

[6] A. Cima, F. Mañosas and J. Villadelprat, Isochronicity for several classes of Hamiltonian systems, J. Differential Equations 157 (1999), 373-413. MR.1713265 (2000h:34073)

[7] W.A. Coppel and L. Gavrilov, The period function of a Hamiltonian quadratic system, Differential Integral Equations 6 (1993), 1357-1365. MR.1235199 (94h:58072)

[8] E. Freire, A. Gasull and A. Guillamon, First derivative of the period function with applications, J. Differential Equations 204 (2004), 139-162. MR2076162 (2005f:37049)

[9] A. Gasull, A. Guillamon and J. Villadelprat, The period function for second-order quadratic ODEs is monotone, Qual. Theory Dyn. Syst. 5 (2004), 201-224. MR2129724 (2005k:34115)

[10] W.S. Loud, Behaviour of the period of solutions of certain plane autonomous systems near centers, Contrib. Differential Equations 3 (1964), 21-36. MR0159985 (28:3199)

[11] P. Mardešić, D. Marín and J. Villadelprat, On the time function of the Dulac map for families of meromorphic vector fields, Nonlinearity 16 (2003), 855-881. MR.1975786 (2004k:37031)

[12] P. Mardešić, D. Marín and J. Villadelprat, The period function of reversible quadratic centers, J. Differential Equations 224 (2006), 120-171. MR2220066 (2006:34076)

[13] F. Rothe, The periods of the Volterra-Lokta system, J. Reine Angew. Math. 355 (1985), 129-138. MR0772486 (86c:92026) 
[14] F. Rothe, Remarks on periods of planar Hamiltonian systems, SIAM J. Math. Anal. 24 (1993), 129-154. MR:1199531 (93m:34058)

[15] C. Rousseau and B. Toni, Local bifurcations of critical periods in the reduced Kukles system, Can. J. Math. 49 (1997), 338-358. MR1447495 (98f:34059)

[16] R. Schaaf, Global behaviour of solution branches for some Neumann problems depending on one or several parameters, J. Reine Angew. Math. 346 (1984), 1-31. MR0727393 (85i:58035)

[17] R. Schaaf, A class of Hamiltonian systems with increasing periods, J. Reine Angew. Math. 363 (1985), 96-109. MR0814016 (87b:58029)

[18] D. Schlomiuk, Algebraic particular integrals, integrability and the problem of the center, Trans. Amer. Math. Soc. 338 (1993), 799-841. MR.1106193 (93j:34037)

[19] J. Smoller and A. Wasserman, Global bifurcation of steady-state solutions, J. Differential Equations 39 (1981), 269-290. MR0607786 (82d:58056)

[20] J. Villadelprat, The period function of the generalized Lotka-Volterra centers, preprint (available online at www.gsd.uab.es).

[21] J. Waldvogel, The period in the Lotka-Volterra system is monotonic, J. Math. Anal. Appl. 114 (1986), 178-184. MR0829122 (87j:92034)

[22] A.A. Zevin and M.A. Pinsky, Monotonicity criteria for an energy-period function in planar Hamiltonian systems, Nonlinearity 14 (2001), 1425-1432. MR.1867085 (2002i:37096)

[23] Y. Zhao, The monotonicity of period function for codimension four quadratic system $Q_{4}$, J. Differential Equations 185 (2002), 370-387. MR1938124 (2003g:34057)

[24] Y. Zhao, The period function for quadratic integrable systems with cubic orbits, J. Math. Anal. Appl. 301 (2005), 295-312. MR2105672 (2005h:34082)

[25] Y. Zhao, On the monotonicity of the period function of a quadratic system, Discrete Contin. Dyn. Syst. 13 (2005), 795-810. MR2153144 (2006d:34072)

[26] H. Żołądek, Quadratic systems with center and their perturbations, J. Differential Equations 109 (1994), 223-273. MR1273302 (95b:34047)

Departament d'Enginyeria Informàtica i Matemàtiques, ETSE, Universitat Rovira i Virgili, 43007 TARRAgOnA, SPAIN 